PS3-47:

Rural Health Research Initiative in the HMORN: A New Scientific Interest Group

Laurel Copeland ${ }^{1}$; Fangfang Sun'; Irina Haller²; Melissa Roberts ${ }^{3}$; Lisa Bailey-Davis ${ }^{4}$; Jeffrey VanWormer ${ }^{5}$; Charles Gessert ${ }^{2}$; Ajay Behl'; Gary Shapiro ${ }^{6}$; Leo Morales ${ }^{7}$; Thomas Elliott ${ }^{2}$

${ }^{1}$ Scott \& White Healthcare; ${ }^{2}$ Essentia Health; ${ }^{3}$ Lovelace Health System; ${ }^{4}$ Geisinger Health System; ${ }^{5}$ Marshfield Clinic / Security Health Plan of Wisconsin; ${ }^{6}$ HealthPartners; ${ }^{7}$ Group Health

Background/Aims: Rural health describes a set of health issues, health care challenges and research priorities driven by a single geo-demographic factor: low population density. Rural areas compared to urbanized areas have fewer providers per capita, longer distance to care, lower socioeconomic status, higher rates of untreated illness, greater exposure to agricultural chemicals, and higher rates of alcohol use, fatal motor vehicle crashes, and suicide. Accessing clinical data for large numbers of rural residents can be challenging. To meet this challenge, seven sites formed the HMORN Rural Health Scientific Interest Group (SIG). Methods: VDW data from seven HMORN sites were analyzed. Rural-Urban Commuting Area (RUCA) codes describe commuting flow but include data on urban, town, and rural tracts; RUCA codes were used to categorize areas as urban, large rural town, small rural town, or isolated rural area. We determined prevalence of chronic conditions by rural status and age group (child, adult, seniors). Results: Common diseases were hypertension, obesity, dyslipidemia, diabetes, alcohol/drug use, depression, and cancers. Most sites saw stable rates of rural vs. urban patients over the years. Rates of pediatric obesity increased at all sites. Adult obesity increased markedly among seniors while dyslipidemia and diabetes increased in all age groups. Cancer among adults also trended upward over time and exceeded national averages. Hypertension among adults appeared lower than US national average in 2010 (32\%). Conclusions: Economic challenges and other factors may further accentuate existing health and health care disparities experienced by many Americans living in rural areas. The Rural Health SIG of the HMORN is poised to conduct meaningful, multi-site research addressing health care issues, health care delivery and care follow-up for this special patient population. Future analyses will explore variation in chronic disease by rural status and the influence of economic factors within geographies.

Keywords: Rural Health; Health Status Disparities; Epidemiology doi:10.3121/cmr.2013.1176.ps3-47

\section{Comparative Effectiveness Research}

A1-1:

"Exercise as a Vital Sign": Impact of Documenting Self-Reported Exercise in Primary Care

Richard Grant $^{1}$; Connie Uratsu ${ }^{1}$; Julie Schmittdiel ${ }^{1}$; Barbara Sternfeld ${ }^{1}$

\section{${ }^{1}$ Kaiser Permanente Northern California}

Background/Aims: Physical inactivity is highly prevalent in US adults and significantly increases mortality risk. We examined the impact of implementing a new primary care visit process to record patient exercise frequency and intensity ("Exercise as a Vital Sign" [EVS]) at the beginning of each visit. Methods: We conducted an observational, quasi-experimental cohort study of 696,267 patients and 1,188 primary care providers to examine the impact of EVS implemented between April 2010 to October 2011 in 4 of 15 regional medical centers. Patients without primary care physicians (PCPs) or with co-morbid conditions that precluded exercise were excluded. We assessed documentation of exercise in PCP progress notes; healthy lifestyle-related referrals (e.g., exercise programs, nutrition and weight loss consultation); and changes in weight or glycemic control in patients with overweight or type 2 diabetes. Results: EVS implementation was associated with greater exercise-related PCP progress note documentation (26.2\% vs. $23.7 \%$ of visits, odds of visit documentation increased 1.12 [95\% CI:1.11-1.13] adjusting for demographic differences and repeated measures); and a small but significant increase in lifestyle-related referrals $(2.1 \%$ vs. 1.7\%; aOR 1.14 [1.11-1.18]), particularly in obese patients (BMI $\geq 30 \mathrm{~kg} / \mathrm{m} 2$;
$4.0 \%$ vs. $3.2 \%$ visits, $P<0.001)$ and patients with type 2 diabetes $(3.5 \%$ vs. $2.6 \%$ visits, $P<0.001)$ compared to visits without EVS. Among patients with at least two BMI measures $(n=633,864)$, patients in EVS medical centers had slight weight loss $(-0.002 \mathrm{~kg} / \mathrm{m} 2)$ whereas control site patients had weight gain $(+0.02 \mathrm{~kg} / \mathrm{m} 2)$. Difference in weight change between facilities favored EVS in linear models controlling for baseline differences $(-0.03 \mathrm{~kg} /$ $\mathrm{m} 2$ [95\% CI: -0.04 to $-0.02 \mathrm{~kg} / \mathrm{m} 2])$. Differences in A1c $(\mathrm{n}=70,083)$ were similarly small but favored EVS facilities (change in A1c: $-0.06 \%$ [95\% CI: -0.08 to $-0.05 \%])$. Conclusions: Exercise data collection during initial patient intake resulted in increased PCP progress note documentation and lifestyle-related referrals. The population-level impact on weight and glycemic control was favorable but slight. We conclude that EVS is a necessary but insufficient first step towards improving the identification and treatment of physical inactivity in primary care.

Keywords: Exercise; Primary Care

doi:10.3121/cmr.2013.1176.a1-1

\section{A1-2:}

Cost-Effectiveness of Interleukin 28B Genotype-Guided Protease Inhibitor Therapy in Treatment-Naïve Patients with Hepatitis C Virus Genotype 2 or 3

Jonathan Bock ${ }^{1}$; Kimberly Fairley' ${ }^{1}$; Robert Smith'; Daniel Maeng${ }^{1}$; James Pitcavage $^{1}$; Nicholas Inverso ${ }^{1}$; Marc Williams ${ }^{1}$

${ }^{1}$ Geisinger Health System

Background/Aims: The addition of protease inhibitors to standard of care (SOC) dramatically increases treatment response in Hepatitis C Virus (HCV) genotype 1 patients. Moreover, Interleukin 28B (IL28B) genotyping helps predict responsiveness for these patients. However, the economic implications of incorporating IL28B genotyping in HCV genotype 2 or 3 infected patients are unknown. This study used a treatment algorithm that included IL28B genotype-guided therapy to examine the short and long-term costeffectiveness of utilizing these single-nucleotide polymorphisms in treatmentnaïve HCV genotype 2 or 3 infected patients. Methods: A treatment algorithm was constructed to reflect a therapy regimen for treatment-naïve patients with $\mathrm{HCV}$ genotype 2 or 3 infection using pegylated-interferon, ribavirin, and telaprevir. To examine the role of the IL28B gene in affecting costs and health outcomes, a decision tree was derived from the treatment algorithm in order to populate a predictive cost model for therapy using our treatment algorithm. Results: Expected short-term costs of therapy following our algorithm were $\$ 21,648.92$ and $\$ 47,972.84$ for the CC and TT genotypes at rs12979860, respectively, and $\$ 47,972.84$ and $\$ 21,648.92$ for patients with the CT genotype at rs12979860 and the TG/GG and TT genotypes at rs8099917, respectively. Predicted costs among patients undergoing SOC therapy were $\$ 20,758.92$. Sustained virologic response (SVR) rates for genotypes $2 / 3$ were predicted to occur in $82.2 \%(8,220$ of 10,000$)$ of patients overall $-88.83 \%(8,883$ of 10,000$)$ and $65.91 \%(6,591$ of 10,000$)$ for the CC and TT genotypes at rs12979860 and $81.01 \%(8,101$ of 10,000$)$ overall for patients with the CT genotype at rs $12979860[72.08 \%(7,208$ of 10,000$)$ and $86.78 \%(8,678$ of 10,000$)$ for the TG/GG and TT genotypes at rs8099917]. Markov modeling predicted a 27.29 quality-adjusted life-expectancy (QALE) after following our treatment algorithm while adding $\$ 7,766.51$ in long-term costs. The model predicted only a 26.65 QALE after SOC therapy (while adding $\$ 9,599.05$ in long-term costs). Conclusions: Although short-term treatment costs of an IL28B genotype-guided approach exceed those of SOC for treatment-naïve $\mathrm{HCV}$ genotype $2 / 3$ infected patients, Markov modeling suggests that lower long-term costs and improved health outcomes may be achieved by the proposed algorithm and provides a dominant cost-effective strategy for treating this population of HCV infected patients.

Keywords: Economics; Cost Modeling; Pharmacogenomics doi:10.3121/cmr.2013.1176.a1-2 